\title{
Independent component analysis based on adaptive artificial bee colony
}

\author{
Shi Zhang ${ }^{1 *}$, Chao-Wei Bao ${ }^{2}$ and Hai-Bin Shen ${ }^{3}$ \\ College of Electrical Engineering, Zhejiang University, Hangzhou, China ${ }^{1}$ \\ Shenzhen State Microelectronics CO., LTD Shenzhen, China ${ }^{2}$ \\ Zhejiang University, Hangzhou, China ${ }^{3}$
}

Received: 12-June-2016; Revised: 25-July-2016; Accepted: 26-July-2016

(C)2016 ACCENTS

\begin{abstract}
Independent component analysis has been more attractive in the signal processing field. An independent component analysis method based on adaptive artificial bee colony algorithm is proposed in this paper, aiming at the problems of slow convergence and low computational precision in existing independent component analysis methods. The algorithm uses the Givens rotation to reduce the amount of variables to be solved. An adaptive global guidance item is introduced in searching strategy to dynamically adjust optimal guiding role. Simulation results show that the adaptive algorithm can separate the linear combinations of sub-Gaussian and super-Gaussian sources successfully and improve the accuracy of separation.
\end{abstract}

\section{Keywords}

Independent component analysis, Artificial bee colony, Adaptive, Search strategy.

\section{Introduction}

Independent component analysis (ICA) is an important branch of blind source separation (BSS). It recovers source signals from receiving mixed based on the source signal's statistical characteristics of independence [1]. ICA becomes more and more important in many areas such as bio-medicine, speech and communications, image processing, earth science, data mining and so on [2].

ICA is mainly composed of the objective function and optimization algorithm. The traditional optimization algorithm uses a natural gradient algorithm (NGA) to optimize the objective function [3]. However, NGA is sensitive to step size, initial value and the selection of nonlinear function. Consequently, it has the shortcomings of narrow applicable range and low accuracy. Artificial bee colony (ABC) algorithm is firstly proposed by professor Karaboga in 2005 [4], and it is a novel algorithm based on swarm intelligence. However, ICA based on ABC, needs to solve $n \times n$ variables. There exists a large amount of calculation in the separation process. Meanwhile, the randomness of search strategy is too strong to control the search direction.

*Author for correspondence

146
Search procedure does not change with the change of the number of iterations. It may lead to low accuracy. In this paper, for the objective function, the Givens rotation was used to reduce the amount of calculation for the optimal strategy, an adaptive strategy was proposed. An adaptive global guidance item was introduced to dynamically adjust optimal guiding role. An adaptive artificial bee colony (AABC) algorithm is proposed to increase the separation accuracy of ICA.

The rest of the paper is organized as follows. Section 1 describes the basic theory of ICA and ABC. Section 2 introduces the proposed algorithm. Section 3 shows simulation results. Finally, Section 4 concludes the paper.

\section{Basic theory}

2.1Independent component analysis

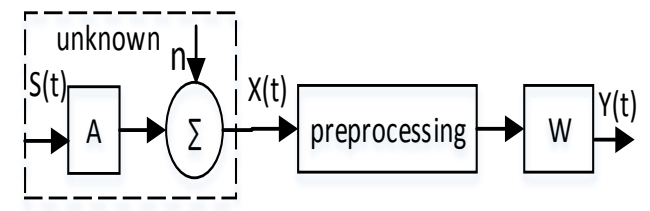

Figure 1 Independent component analysis model

The basic ICA model is depicted as Figure 1 . 


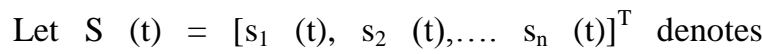
independent source signal vector that comes from $n$ signal sources. Ignoring the additive Gaussian noise and assuming $\mathrm{m}=\mathrm{n}$, we can get observed mixture $\mathrm{X}$ $(\mathrm{t})=\left[\mathrm{x}_{1}(\mathrm{t}), \mathrm{x}_{2}(\mathrm{t}), \ldots \mathrm{x}_{\mathrm{n}}(\mathrm{t})\right]^{\mathrm{T}}$ under the circumstances of instantaneous linear mixture. Consider the following:

$$
\mathbf{X}(t)=\mathbf{A S}(t)
$$

Where $\mathbf{A} \in \mathbf{R}^{n \times n}$ is a $n \times n$ non-singular constant matrix. After preprocessing, optimization algorithm is used to solve the objective function to find the orthogonal separating matrix W. Then estimated source signals can be separated:

$$
\mathbf{Y}(t)=\mathbf{W X}(t)
$$

Kurtosis is the classical measure of independence. The objective function can be defined as [5]:

$$
\mathbf{I}(\mathbf{Y})=-\sum_{\mathrm{i}=1}^{\mathrm{N}}\left|\operatorname{kurt}\left(\mathrm{Y}_{\mathrm{i}}\right)\right|=-\sum_{\mathrm{i}=1}^{\mathrm{N}}\left|\frac{E\left(\mathrm{Y}_{\mathrm{i}}^{4}\right)}{E^{2}\left(\mathrm{Y}_{\mathrm{i}}^{2}\right)}-3\right|
$$

Where kurt $\left(\mathrm{Y}_{\mathrm{i}}\right)$ is the kurtosis of separated signals. The dependence among the components is minimized when $\mathbf{I}(\mathbf{Y})$ is minimized for a separating matrix W. Separation performance can be evaluated by PI (Performance Index, PI) [1]:

$$
\mathbf{P}=\frac{1}{n(n-1)} \sum_{i=1}^{n}\left[\left(\sum_{k=1}^{n} \frac{\left|c_{i k}\right|}{\max _{j}\left(\left|c_{i j}\right|\right)}-1\right)+\left(\sum_{k=1}^{n} \frac{\left|c_{k i}\right|}{\max _{j}\left(\left|c_{j i}\right|\right)}-1\right)\right]
$$

Where $\mathbf{C}=\mathbf{W A}$ is the global matrix and $\mathrm{c}$ being the element of matrix $\mathrm{C}$. The more PI reaches zero, the better separation performance is.

\subsection{Basic artificial bee colony algorithm}

Bee colony is composed of employed bees, onlooker bees and scout bees. The process of searching for the best food for the bee colony represents the process of finding the best solution of the objective function. $\mathrm{ABC}$ algorithm is made up of four stages: initialization stage, employed bee stage, onlooker bee stage and scout bee stage $[6,7]$.

At the first initialization stage, each food source is initialized as follows:

$$
\Theta_{\mathrm{ij}}=\theta_{\min }+\operatorname{rand}(0,1)\left(\theta_{\max }-\theta_{\min }\right)
$$

where $_{i} \in\{1,2, \ldots, \quad \mathrm{SN}\}, \mathrm{j} \in\{1,2, \ldots, \mathrm{D}\}, \Theta_{\mathrm{i} j}$ represents the $j$ th dimension of $i$ th food source in the colony. rand $(0,1)$ is a random number between [0,1]. $\theta_{\max }$ and $\theta_{\min }$ are upper and lower limit values[8-10].
On the second employed bee stage, a candidate food position is produced from the old one and then evaluated. The ABC uses the following search strategy:

$$
\Theta_{\mathrm{ij}}=\theta_{\mathrm{ij}}+\varphi_{\mathrm{ij}}\left(\theta_{\mathrm{ij}}-\theta_{\mathrm{kj}}\right)
$$

$\mathrm{k} \in\{1,2, \ldots, \quad \mathrm{SN}\}, i \neq k$, where $\mathrm{j}$ and $\mathrm{k}$ are randomly chosen indexes. $\varphi_{\mathrm{ij}}$ is a random number between $[-1,1] . \quad \Theta_{i j}$ and $\theta_{i j}$ represent the $j$ th dimension of $i$ th new and old food source in the colony. After each candidate source position is produced, a greedy selection mechanism is employed as the selection operation between the old one and the candidate one. If the new food has equal or better fitness than the old one, it replaces the old one in the memory. Otherwise, the old one is retained. The fitness is calculated by the following expression [11]:

$F\left(\Theta_{i}\right)= \begin{cases}1+\left|J\left(\Theta_{i}\right)\right|, & J\left(\Theta_{i}\right)<0 \\ 1 / 1+J\left(\Theta_{i}\right), & J\left(\Theta_{i}\right) \geq 0\end{cases}$

Where $F\left(\Theta_{i}\right)$ is the fitness value of the solution $\Theta_{i}$ evaluated by its employed bee. $J\left(\Theta_{i}\right)$ is the value of the objective function. The maximum of fitness is corresponding to the minimum of the objective function.

At the third onlooker bee stage, an onlooker bee chooses a source food of the employed bee depending on the probability value $\mathrm{P}\left(\Theta_{i}\right)$. The $\mathrm{ABC}$ uses the following selection strategy:

$$
P\left(\Theta_{i}\right)=F\left(\Theta_{i}\right) / \sum_{i=1}^{S N} F\left(\Theta_{i}\right)
$$

Where $\mathrm{P}\left(\Theta_{\mathrm{i}}\right)$ is the selected probability value of the $i$ th source food. After the source food is chosen, the ABC uses the expression (6) to search in the neighborhood and chose the better source food [1213].

On the fourth scout bee stage, if a source food cannot be improved further through a predetermined number of cycles called limit, the source food will be abandoned. The scout bee will produce a new food source randomly according to equation (5).

\section{ICA based on adaptive artificial bee colony}

3.1Givens rotation

According to the related research papers, the separating matrix is orthogonal. These papers orthogonalize the separating matrix after being calculated. In this way, $n \times n$ variables need to be 
calculated. This method exists large amount of calculation in the separation process. According to the relevant papers, the degrees of freedom of $n$ dimensional orthogonal matrix are $n \times(n-1) / 2$. In other words, we can parameterize the orthogonal matrix with $\mathrm{n} \times(\mathrm{n}-1) / 2$ variables. $\mathrm{n} \times(\mathrm{n}-1) / 2$ Givens planar rotations are used to parameterize the $\mathbf{n} \times \mathbf{n}$ orthogonal matrix [8]. When $\mathrm{n}=3$, the dimensionality of the solution of the objective function before dimensionality reduction is 9 . The solution can be expressed by

$$
\theta=\left[\theta_{1}, \theta_{2}, \theta_{3}, \theta_{4}, \theta_{5}, \theta_{6}, \theta_{7}, \theta_{8}, \theta_{9}\right] \text {. After }
$$

adopting the Givens rotation, the dimensionality is 3 , and the solution can be expressed by

$\theta=\left[\theta_{1}, \theta_{2}, \theta_{3}\right]$. The separating orthogonal matrix can be expressed as follows:

$$
\begin{aligned}
& W=\left[\begin{array}{ccc}
1 & 0 & 0 \\
0 & \cos \theta_{1} & -\sin \theta_{1} \\
0 & \sin \theta_{1} & \cos \theta_{1}
\end{array}\right]\left[\begin{array}{ccc}
\cos \theta_{2} & 0 & -\sin \theta_{2} \\
0 & 1 & 0 \\
\sin \theta_{2} & 0 & \cos \theta_{2}
\end{array}\right]\left[\begin{array}{ccc}
\cos \theta_{3} & -\sin \theta_{3} & 0 \\
\sin \theta_{3} & \cos \theta_{3} & 0 \\
0 & 0 & 1
\end{array}\right] \\
& \theta_{1}, \theta_{2}, \theta_{3} \in[0,2 \pi] 。
\end{aligned}
$$

For the solution of the objective function, the Givens rotation is used to reduce the amount of calculation.

\subsection{Modified $\mathrm{ABC}$ search strategy}

Equation (6) shows the search strategy of $\mathrm{ABC}$. There are two disadvantages of the strategy. On the one hand, the search direction is random and the search is the blind lack of guidance. The parameter of the step size $\varphi_{i j}$ is a random number in the range [-

$1,1]$. And the neighbourhood bee $\theta_{\mathrm{kj}}$ is also chosen randomly; on the other hand, the strategy is fixed without changing with the change of the iteration number. In other words, the search strategy has strong ability to explore the global scope, which can avoid to fall into the local extreme value; but it has a weak ability to exploit the best source food in the local range. In order to solve the first problem, a global guidance item $\Theta_{\text {best }}$ is introduced in search strategy to guide the search direction and the step size. To solve the second problem, adaptive coefficient $g_{i j}$ is introduced to change with the change of the iteration number iter. All in all, the adaptive guidance item $g_{i j}\left(\Theta_{\text {best } j}-\theta_{i j}\right)$ is introduced. And the coefficient of the step size $\Phi_{i j}$ changes adaptively. The modified search strategy is devised as follows:

$$
\begin{aligned}
& \Theta_{\mathrm{ij}}=\theta_{\mathrm{ij}}+\Phi_{\mathrm{ij}}\left(\theta_{\mathrm{ij}}-\theta_{\mathrm{kj}}\right)+g_{i j}\left(\Theta_{\text {bestj }}-\theta_{i j}\right) \\
& \Phi_{i j}=\left\{\begin{array}{l}
b_{i j}\left(\frac{\mathrm{J}\left(\Theta_{\text {best }}\right)-\mathrm{J}\left(\Theta_{\mathrm{i}}\right)}{\mathrm{J}\left(\Theta_{\text {best }}\right)-\mathrm{J}\left(\Theta_{\mathrm{k}}\right)}\right), \mathrm{J}\left(\Theta_{\mathrm{k}}\right) \neq \mathrm{J}\left(\Theta_{\text {best }}\right) \\
\varphi_{i j}, \quad \mathrm{~J}\left(\Theta_{\mathrm{k}}\right)=\mathrm{J}\left(\Theta_{\text {best }}\right)
\end{array}\right. \\
& g_{i j}=2 /\left(\exp \left(-g 1\left(\frac{\text { iter }}{\text { iter }_{\text {max }}}\right)^{g 2}\right)+1\right)-1
\end{aligned}
$$

Where $\Theta_{\text {best } j}$ is the $j$ th dimension of the best solution. $b_{i j}$ is +1 or -1 randomly. $\mathbf{J}\left(\Theta_{i}\right)$ is the value of the objective function. iter represents the current iteration number and iter $_{\max }$ represents the maximum cycle number. $g 1$ and $g 2$ are constants. The adaptive step size coefficient $\Phi_{\mathrm{ij}}$ can adjust step length adaptively: at the beginning of the iteration, there is large difference between the value of $\mathbf{J}\left(\Theta_{\text {best }}\right)$ and $\mathbf{J}\left(\Theta_{\mathrm{i}}\right)$. According to the formula, the modified search strategy has a large step size and wide search range; at the later period of iteration, the values of $\mathbf{J}\left(\Theta_{\text {best }}\right)$ and $\mathbf{J}\left(\Theta_{i}\right)$ are close.

Therefore, the step size coefficient is small and close to zero, which can accelerate the finding the best solution.

The adaptive guidance item coefficient $\boldsymbol{g}_{i j}$ can adjust the best solution item $\Theta_{\text {bestj }}-\theta_{i j}$ : at the beginning of the iteration, the value of $g_{i j}$ is small to weaken the item's searching capability; at the later period of iteration, the value is large to strengthen the item's searching capability, which can accelerate the finding the best solution. Therefore, the adaptive search strategy can adjust the step size and the best solution guidance capability, which can balance the global exploration and locaproprol exploitation.

\subsection{Flow of ICA based on AABC}

ICA is mainly composed of objective function and optimization algorithm. This paper uses the expression (3) as the objective function, and $\mathrm{AABC}$ algorithm is used as the optimization algorithm.

\section{Experimental results}

\subsection{Source signals}


To illustrate the performance of the proposed ICA algorithm based on $\mathrm{AABC}$, three signals are used as source signals. The source signals have different Kurtosis: one is super-Gaussian signal and others are sub-Gaussian signals.

$\mathrm{s}_{1}(\mathrm{t})=((\bmod (\mathrm{t}, 2 \mathrm{D})-100) / 100)^{5}$;

$\mathrm{s}_{2}(t)=\sin (0.018 \pi t)$

$s_{3}(t)=\sin (0.0018 \pi t) \sin (0.06 \pi t)$

The values of Kurtosis for the source signals are $2.8500,-1.5000$ and -0.7130 . The elements of the $3 * 3$ mixing matrix $A$ are random numbers distributed uniformly in the range $[-1,1]$.

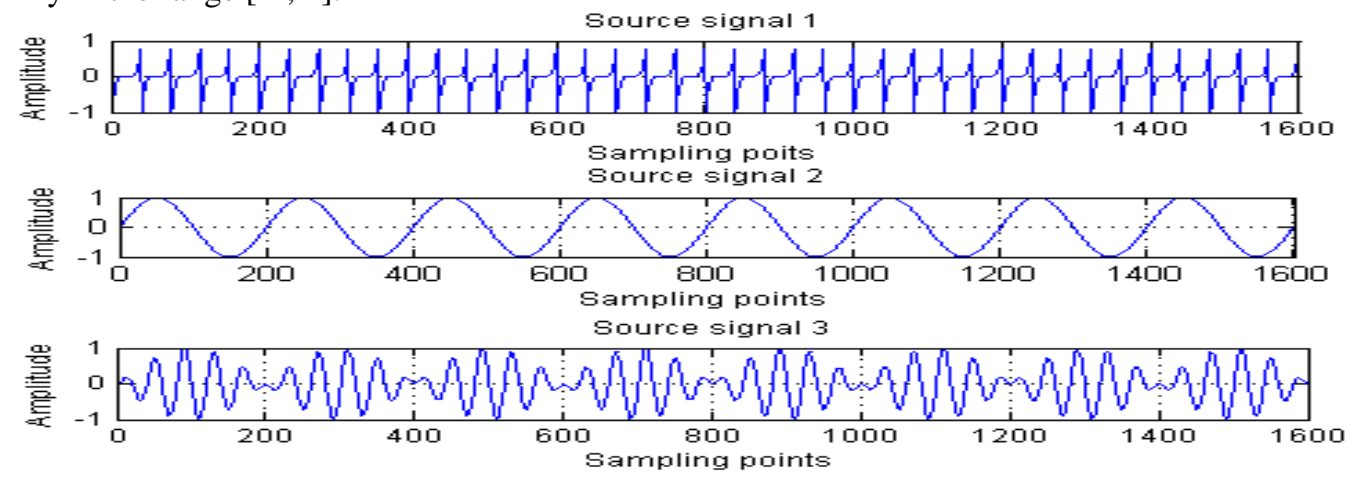

Figure 2 Original source signals

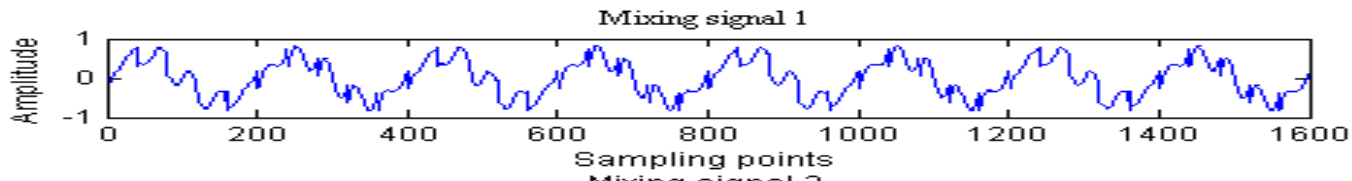

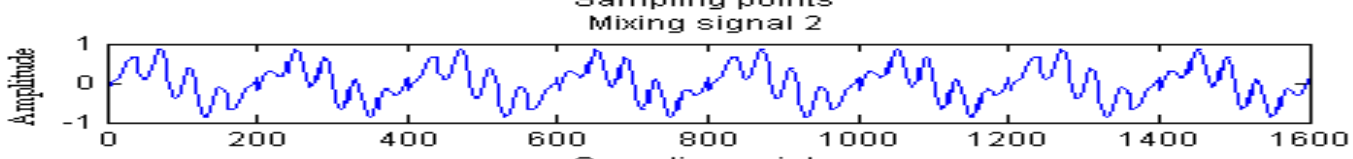

Sampling points

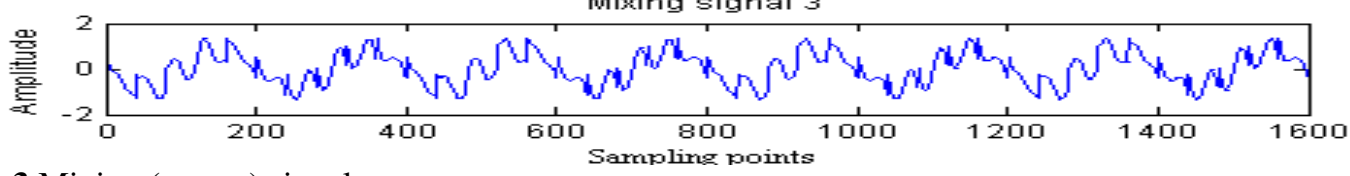

Figure 3 Mixing (sensor) signals

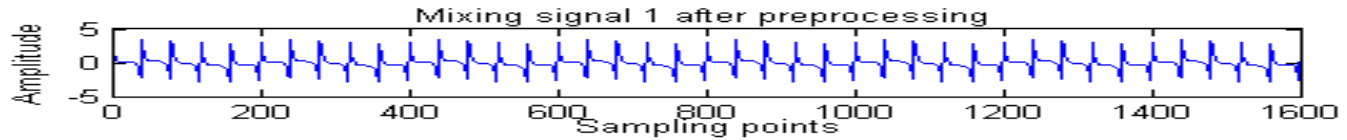

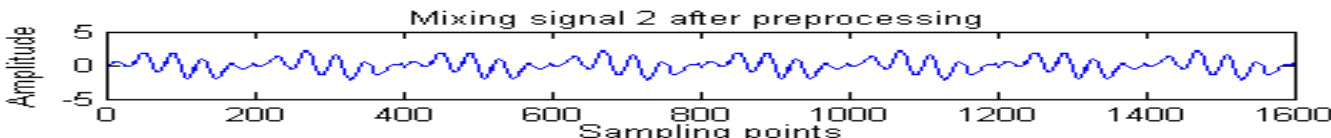

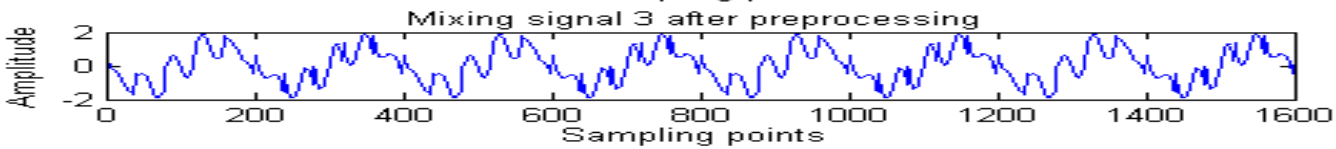

Figure 4 Mixing signals after preprocessing 


\subsection{Performance measure}

This paper compares the AABC algorithm with two other ICA algorithms, which are named NGA and $\mathrm{ABC}$. They are evaluated using the performance index presented in expression (4).

The initialization values of the parameters for NGA are set as $\mu=0.0005$, iter $\max _{\max }=1000$; and the initialization values of the parameters for $\mathrm{ABC}$ are summarized in Table 1.

The common parameters between $\mathrm{AABC}$ and $\mathrm{ABC}$ are illustrated in Table 1, and others are set as $\mathrm{g} 1=50$, $\mathrm{g} 2=6$.

Table1 Parameters of ABC

\begin{tabular}{ll}
\hline ABC Parameters & Value \\
\hline Number of employed bees & 15 \\
Number of onlooker bees & 15 \\
Number of scout bees & 1 \\
The parameter of limit & 90 \\
Number of maximum cycles & 1000 \\
\hline
\end{tabular}

Firstly, this paper will evaluate the performance of $\mathrm{NGA}, \mathrm{ABC}$ and $\mathrm{AABC}$ using wave form. Separation signals of NGA, $\mathrm{ABC}$ and $\mathrm{AABC}$ are shown in Figure 5.

As Figure 5 shows, the order of separation signals is not fixed and so is the amplitude. The separation quality can be evaluated qualitatively from Figure 5 . The separation performance of NGA is obviously very poor, and the signals are seriously deformed. However, $\mathrm{ABC}$ and $\mathrm{AABC}$ can recover superGaussian and sub-Gaussian signals successfully. Therefore, $\mathrm{ABC}$ and $\mathrm{AABC}$ can separate signals of different Kurtosis.

Performance index (PI) given by the expression (4) can be used to evaluate the separation performance quantitatively. Each of the experiments for the three algorithms was repeated 50 times.

The maximum, minimum and average of PI obtained by NGA, ABC and AABC have been recorded in Table 2. Moreover, Figure 6 shows the convergence curves of mean PI for different separation algorithms.

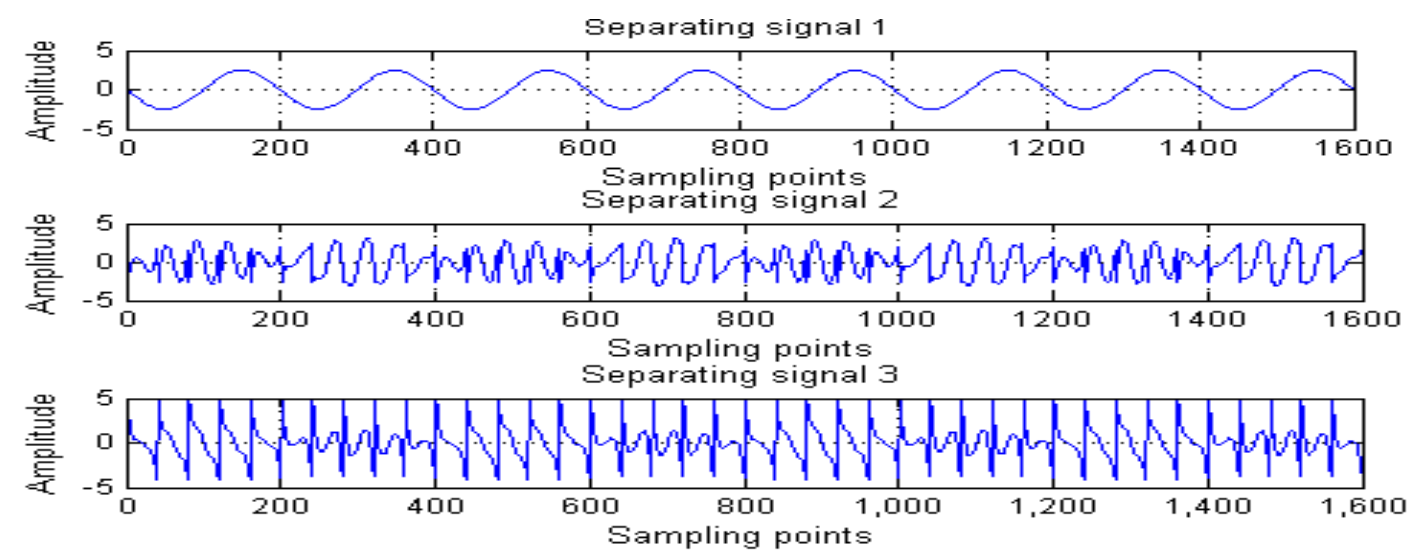

(a) Separating signals of NGA
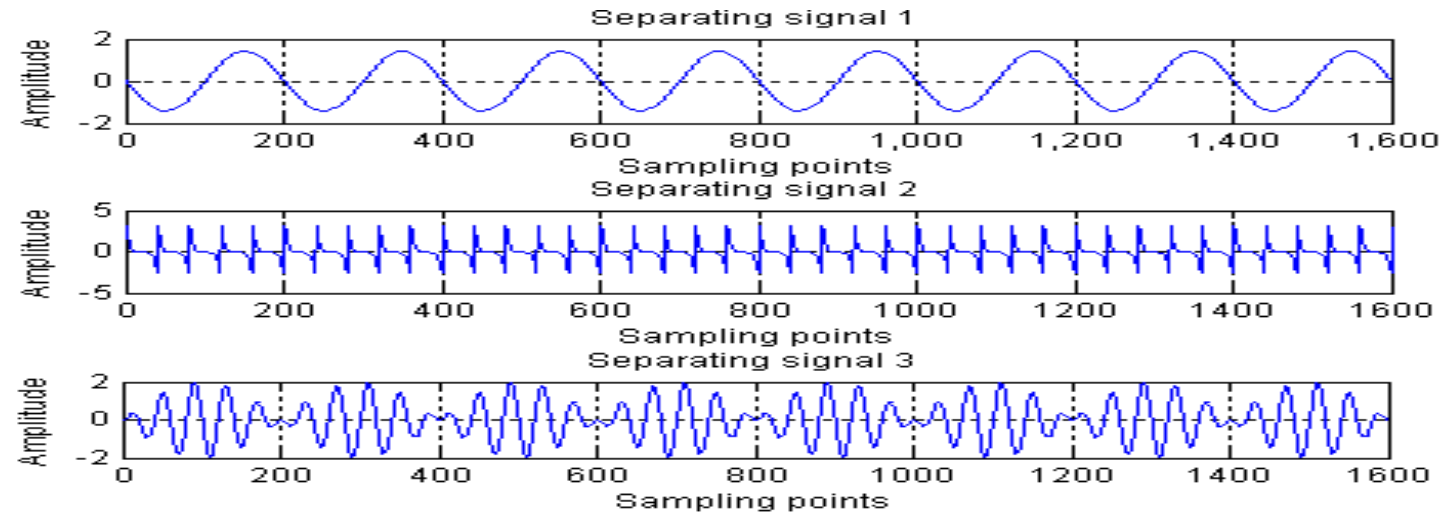

(b) Separating signals of $\mathrm{ABC}$ 

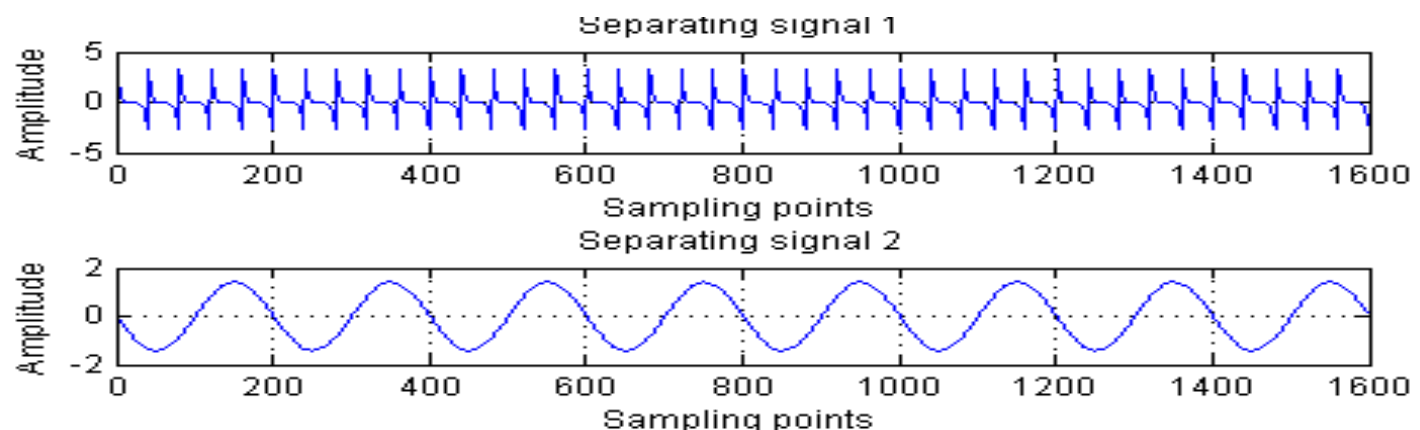

Separating signal

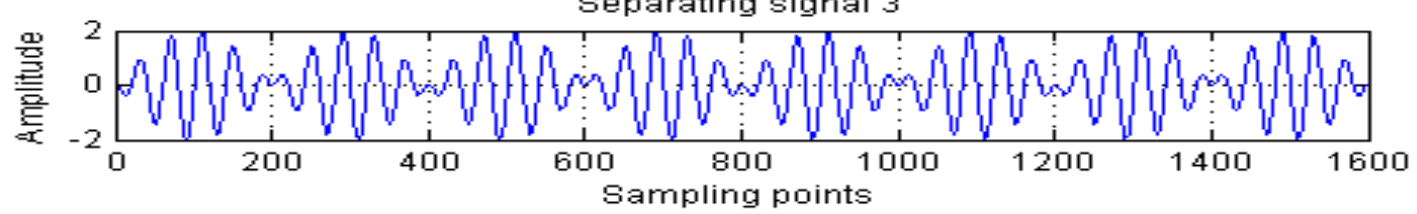

(c) Separating signals of AABC

Figure 5 Separating signals of NGA, ABC and AABC

Table2 Performance index

\begin{tabular}{ccccc}
\hline Algorithm & \multicolumn{3}{c}{ Performance index PI } \\
\cline { 2 - 5 } & Minimum & Maximum & Average & Standard Deviation \\
NGA & 0.34527 & 0.40063 & 0.37858 & 0.0279 \\
ABC & $1.4803 \mathrm{e}-16$ & $2.0084 \mathrm{e}-07$ & $2.7025 \mathrm{e}-08$ & $6.3057 \mathrm{e}-08$ \\
AABC & $7.4015 \mathrm{e}-17$ & $2.5905 \mathrm{e}-15$ & $1.0362 \mathrm{e}-15$ & $8.6738 \mathrm{e}-16$ \\
\hline
\end{tabular}

As seen from the results in Table 2, the convergence precision of $\mathrm{AABC}$ algorithm is evidently better than $\mathrm{ABC}$ and NGA, from minimum value, maximum value or average value. Quantitatively, the separation accuracy of $\mathrm{AABC}$ is improved to about three orders of magnitude than $\mathrm{ABC}$. In other words, ICA based on $\mathrm{AABC}$ performs better than NGA and $\mathrm{ABC}$, and the convergence precision was improved obviously. The average convergence trend of PI was showed in Figure 6 NGA converges to minimum value early and has low convergence accuracy.
$\mathrm{ABC}$ algorithm converges to minimum value at about 100 iteration number. At the beginning of the iteration, the curve of $\mathrm{AABC}$ is similar to $\mathrm{ABC}$. As the number of iteration increases, the best solution guidance item of the modified search strategy plays a more and more important role, which can accelerate the finding the best solution and improve the convergence accuracy.

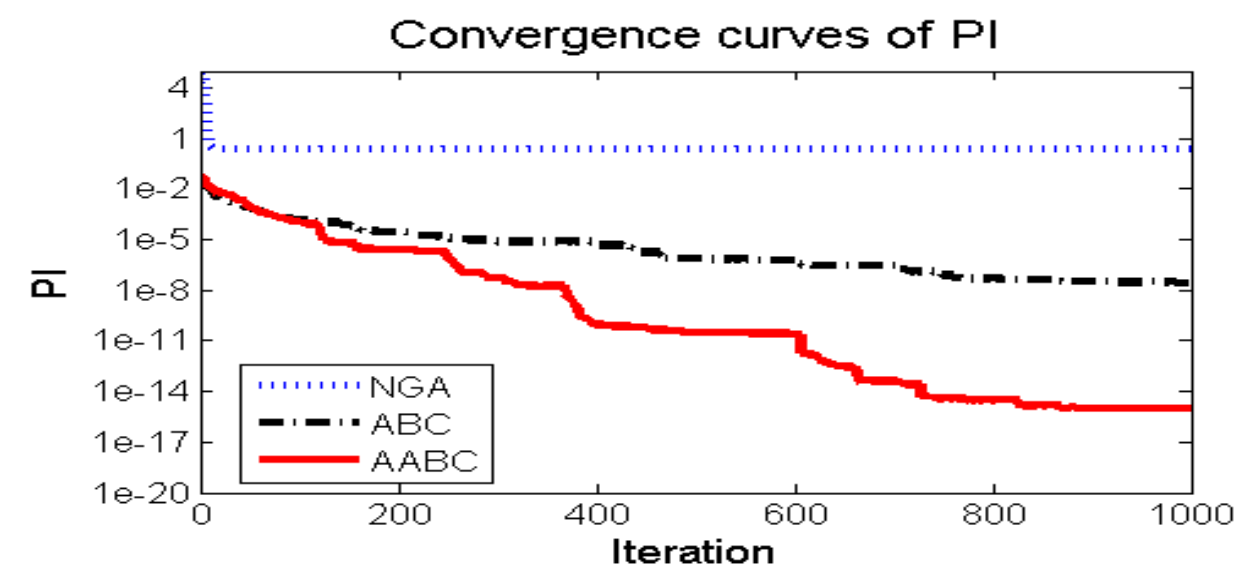

Figure 6 Convergence curves of PI 


\section{Conclusion}

Independent component analysis (ICA) has seen increasing demand nowadays in many areas of signal processing. In this paper, Aiming at reducing the amount of calculation, the Givens rotation is used to reduce the number of variables. Moreover, the adaptive search strategy is introduced to adjust the step size and the best solution guidance capability, which can balance the global exploration and local exploitation. In other words, AABC algorithm is proposed to improve the ICA separation performance. The results of the experiments have shown its success in separating the linear combinations of sub-Gaussian and super-Gaussian sources. And the separation accuracy is improved.

\section{Acknowledgment}

None.

\section{Conflicts of interest}

The authors have no conflicts of interest to declare.

\section{References}

[1] Lee TW. Independent component analysis. In independent component analysis 1998 (pp. 27-66). Springer.

[2] Comon P, Jutten C, editors. Handbook of blind source separation: independent component analysis and applications. Academic press; 2010.

[3] Liu JQ, Feng DZ, Zhang WW. Adaptive improved natural gradient algorithm for blind source separation. Neural Computation. 2009; 21(3):872-89.

[4] Karaboga D. An idea based on honey bee swarm for numerical optimization. Technical report-tr06, Erciyes University, engineering faculty, computer engineering department; 2005.

[5] Ebrahimzadeh A, Mavaddati S. A novel technique for blind source separation using bees colony algorithm and efficient cost functions. Swarm and Evolutionary Computation. 2014:15-20.

[6] Karaboga D, Basturk B. A powerful and efficient algorithm for numerical function optimization: artificial bee colony (ABC) algorithm. Journal of Global Optimization. 2007; 39(3):459-71.

[7] Karaboga D, Basturk B. On the performance of artificial bee colony (ABC) algorithm. Applied Soft Computing. 2008; 8(1):687-97.

[8] Mladenova CD, Mladenov IM, Todorov MD. About parametric representations of SO (n) matrices and plane rotations. In AIP conference proceedingsAmerican institute of physics 2012 (pp. 280-7).

[9] Wang J, Yao Y, Mao Y, Sheng B, Mi N. Fresh: fair and efficient slot configuration and scheduling for hadoop clusters. In IEEE 7th International conference on cloud computing 2014 (pp. 761-8). IEEE.

[10] Bawa RK, Sharma G. Modified min-min heuristic for job scheduling based on QoS in grid environment. In international conference on information management in the knowledge economy (IMKE) 2013 (pp. 16671). IEEE.

[11] Chen H, Wang F, Helian N, Akanmu G. User-priority guided min-min scheduling algorithm for load balancing in cloud computing. In national conference on parallel computing technologies 2013 (pp. 1-8). IEEE.

[12] Diaz CO, Pecero JE, Bouvry P. Scalable, low complexity, and fast greedy scheduling heuristics for highly heterogeneous distributed computing systems. The Journal of Supercomputing. 2014; 67(3):837-53.

[13] Huang SC, Jiau MK, Lin CH. A genetic-algorithmbased approach to solve carpool service problems in cloud computing. IEEE Transactions on Intelligent Transportation Systems. 2015; 16(1):352-64.
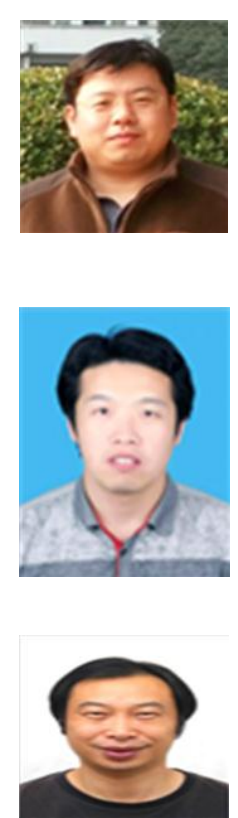

Shi Zhang is a doctoral student at the Institute of electrical engineering, Zhejiang University. His research direction is intelligent security and chip design.

Email: oec_ljw2009@163.com

Chao-Wei Bao, is a doctoral student at the Institute of electrical engineering, Zhejiang University, 2014. He is now the technical director of Shenzhen State Microelectronics CO.,LTD, his research direction is programmable logic devices design technology and safety protection technology.

Hai-Bin Shen, is a professor and doctoral supervisor of Zhejiang University, his research direction is intelligent security and chip design. 